\title{
Systemic Immune-Inflammation Index Predicts Prognosis of Patients With Esophageal Squamous Cell Carcinoma Undergoing Radical Radiotherapy: A Propensity Score-Matched Analysis
}

\section{Yan Zhao}

Hebei Medical University Fourth Affiliated Hospital and Hebei Provincial Tumor Hospital https://orcid.org/0000-0002-84082458

\section{Wen-Bin Shen}

Department of Radiation Oncology, Fourth Affiliated Hospital of Hebei Medical University, Shijiazhuang, Hebei, 050011, China Chun-Yang Song

Department of Radiation Oncology, Fourth Affiliated Hospital of Hebei Medical University, Shijiazhuang, Hebei, 050011, China

Jin-Rui Xu

Department of Radiation Oncology, Fourth Affiliated Hospital of Hebei Medical University, Shijiazhuang, Hebei, 050011, China

\section{Shu-Guang Li}

Department of Radiation Oncology, Fourth Affiliated Hospital of Hebei Medical University, Shijiazhuang, Hebei, 050011, China

\section{Wen-Zhao Deng}

Department of Radiation Oncology, Fourth Affiliated Hospital of Hebei Medical University, Shijiazhuang, Hebei, 050011, China

\section{Juan Li}

Department of Radiation Oncology, Fourth Affiliated Hospital of Hebei Medical University, Shijiazhuang, Hebei, 050011, China

Shu-Chai Zhu ( $\nabla$ sczhu1965@163.com )

Department of Radiation Oncology, Fourth Affiliated Hospital of Hebei Medical University, Shijiazhuang, Hebei, 050011, China

\section{Research}

Keywords: Systemic immune-inflammation index, esophageal squamous cell carcinoma, radiotherapy, prognosis

Posted Date: December 16th, 2020

DOI: https://doi.org/10.21203/rs.3.rs-126794/v1

License: (1) This work is licensed under a Creative Commons Attribution 4.0 International License. Read Full License 


\section{Abstract}

\section{Background}

The systemic immune-inflammation index (SII) was recently investigated as a prognostic predictor in several kinds of solid tumors, including esophageal squamous cell carcinoma (ESCC) after esophagectomy. However, just few studies regarding SII in patients with ESCC undergoing radical radiotherapy are available. In addition, there has been hardly any report investigating the change trend of SII during radiotherapy. The aim of this study was to identify the prognostic value of SII in ESCC patients undergoing radical radiotherapy.

Methods

We retrospectively reviewed 303 ESCC patients undergoing radical radiotherapy. The change trend of SII was assessed by the box plot and curve fitting method. The time-dependent receiver operating characteristics was used to determine the optimal cutoff value of the SII. The association between SII and survival was determined by Kaplan-Meier method and Cox regression model. Propensity score matching (PSM) was applied to imbalance the baseline characteristics.

Results

High SIl was associated with poor overall survival (OS) and progression-free survival (PFS) in patients with ESCC undergoing radical radiotherapy. Multivariate analysis showed that SII was a significant predictor for OS and PFS, whether before or after PSM. In addition, SII displayed an exponential increase trend during radiotherapy. The change of SII was also associated with OS and PFS.

Conclusions

The SII is a significant and independent predictor for OS and PFS of ESCC patients undergoing radical radiotherapy. Based on simple and inexpensive standard laboratory measurements, SIl can be a promising marker for ESCC patients.

\section{Introduction}

Esophageal cancer is the eighth most common cancer worldwide in incidence and the sixth most common cause in mortality [1]. Esophageal squamous cell carcinoma (ESCC) is the most common pathological type in China, accounting for $90 \%$ of cases [2]. Although multidisciplinary treatment is commonly applied, including surgery combined with radiotherapy and chemotherapy, the 5-year overall survival remains poor. Therefore, assessing dependable prognostic predictors in ESCC remains very important.

During the past decade, there has been an increasing interest in the associations between systemic inflammatory response, immune status and long-term outcomes of several malignancies for many clinical researchers [3-7]. Some inflammatory and immune response biomarkers, such as platelet-lymphocyte ratio (PLR), neutrophil-lymphocyte ratio (NLR), and monocytelymphocyte ratio (MLR), have been demonstrated to be associated with postoperative prognosis in various cancers, including esophageal cancer, lung cancer, gastric cancer, et al [8-11]. However, these inflammatory biomarkers only integrate two kinds of cells. Recently, a novel indicator, systemic immune-inflammation index (SII), based on lymphocyte, neutrophil and platelet counts, has been proved as a powerful prognostic indicator for survival in various types of cancers [12-15]. Some previous studies have showed that preoperative SII is a prognostic marker for esophageal cancer undergoing esophagectomy [16-20]. However, to our best knowledge, just few studies regarding SII in patients with ESCC undergoing radical radiotherapy are available. In addition, there has been hardly any report investigating the change trend of SII during radiotherapy. Thus, the aim of this study was to investigate the prognostic value of SII in predicting survival in ESCC patients undergoing radical radiotherapy. Meanwhile, the change trend of SII during radiotherapy was analyzed. To reduce biases due to the different distributions of co-variables among the comparable groups and to increase statistical power, propensity score matching (PSM) was applied.

\section{Materials And Methods}

\section{Patients}


This retrospective analysis was conducted in 303 patients with ESCC who underwent radical radiotherapy from January 2011 to December 2017 at Fourth Hospital of Hebei Medical University in Shijiazhuang, China. All of the patients enrolled in the analysis met the following inclusion criteria: ESCC confirmed by histopathology; Karnofsky score $\geq 70$ points; neither chemotherapy nor other anti-tumor therapy before radiotherapy; no history of malignant disease. All subjects gave their written informed consent to the study protocol, which was approved by the ethics committee of Fourth Hospital of Hebei Medical University, Shijiazhuang, China. According to TNM stage system issued by the American Joint Committee on cancer (AJCC; 7th edition), 30 patients with Stage I, 118 patients with Stage II and 155 patients with Stage III were included in this study. The characteristics of the patients are summarized in Table 1.

\section{Treatment}

In this study, all patients received intensity-modulated radiotherapy, including 180 patients undergoing involved-field irradiation and 123 patients undergoing elective node irradiation. The gross tumor volume (GTV), clinical tumor volume, and planned tumor volume were outlined in the RT treatment planning system, according to computed tomography simulation scan, barium esophagram, electronic gastroscopy and/or computed tomography-positron emission tomography. The primary tumor and positive regional lymph nodes were defined as GTV. The prescribed dose was set at 54-68 Gy (in 27-34 fractions over a range of 57 weeks). The elective node region was irradiated at the prescribed dose of 46-52 Gy. The patients undergoing adjuvant chemotherapy mainly received cisplatin combined with paclitaxel or 5 -fluorouracil for 1 to 6 cycles.

\section{Calculation of SII}

SII = platelet counts $\times$ neutrophil counts/ lymphocyte counts. The optimal cutoff value for SIl was calculated using the timedependent receiver operating characteristics (ROC).

\section{Follow-up}

At our institution, follow-up examinations were performed every 3-month intervals in the first 2 years, then 6-month intervals. The routine examination included physical examination, blood tests, biological investigations, tumor markers, thoracic CT scanning, and barium esophagram.

\section{Statistical analysis}

All recorded data were analyzed using SPSS (version 23.0, IBM Corporation, Armonk, NY, USA). The change trend of SIl was assessed by the box plot and curve fitting method. Pearson chi-square test was used to assess the correlation between different categorical variables. The OS rate was calculated using the Kaplan-Meier method, and a log-rank test was used to assess survival differences between groups. Cox proportional hazards regression analysis was performed to identify independent variables. PSM was carried out to reduce biases due to the different distributions of co-variables among the comparable groups. $\mathrm{P}$ values $<0.05$ indicated statistical significance.

\section{Results}

\section{Correlation between SII and clinicopathological characteristics before and after propensity score matching}

The optimal cutoff value for SIl levels was set at 586 in this study, based on the OS (sensitivity: 60.5\%; specificity: 56.9\%; AUC of the ROC curve: 0.602; $P=0.012$ ). Based on their SII values, patients were categorized as high SIl group (more than 586 ) or low SII group (586 or less).

The characteristics of 303 ESCC patients undergoing radical radiotherapy are presented in Table 1. Among the 303 patients, 178 (58.7\%) were male and 125 (41.3\%) were female. The median age was 67 years (range, $41-90$ years). Patients with high SII in complete databases were more likely to be middle cancer $(P=0.010)$, advanced T stage $(P=0.001)$ and TNM stage $(P=0.006)$.

Table 1 The Correlation between SII and characteristics in 303 ESCC patients before and after PSM 


\begin{tabular}{|c|c|c|c|c|c|c|c|c|}
\hline \multirow[t]{2}{*}{ Characteristics } & \multirow{2}{*}{$\begin{array}{l}\text { Total } \\
\text { patients }\end{array}$} & \multicolumn{3}{|c|}{ Before PSM $(n=303)$} & \multirow{2}{*}{$\begin{array}{l}\text { Total } \\
\text { patients }\end{array}$} & \multicolumn{3}{|c|}{ After PSM ( $n=222)$} \\
\hline & & $\begin{array}{l}\text { SII } \leq 586 \\
(n=131)\end{array}$ & $\begin{array}{l}\text { SII>586 } \\
(n=172)\end{array}$ & $P$ & & $\begin{array}{l}\text { SII } \leq 586 \\
(n=111)\end{array}$ & $\begin{array}{l}S \|>586 \\
(n=111)\end{array}$ & $P$ \\
\hline Sex & & & & 0.235 & & & & 0.130 \\
\hline Male & 178 & 82 & 96 & & 135 & 73 & 62 & \\
\hline Female & 125 & 49 & 76 & & 87 & 38 & 49 & \\
\hline Age & & & & 0.493 & & & & 0.415 \\
\hline$\leq 65$ & 132 & 60 & 72 & & 94 & 50 & 44 & \\
\hline$>65$ & 171 & 71 & 100 & & 128 & 61 & 67 & \\
\hline $\begin{array}{l}\text { Tumor } \\
\text { location }\end{array}$ & & & & 0.010 & & & & 0.271 \\
\hline Cervical+Upper & 101 & 54 & 47 & & 59 & 34 & 25 & \\
\hline Middle & 135 & 46 & 89 & & 103 & 46 & 57 & \\
\hline Lower & 67 & 31 & 36 & & 60 & 31 & 29 & \\
\hline T stage & & & & 0.001 & & & & 0.296 \\
\hline $\mathrm{T} 1+\mathrm{T} 2$ & 83 & 47 & 36 & & 61 & 27 & 34 & \\
\hline T3 & 103 & 48 & 55 & & 85 & 48 & 37 & \\
\hline T4 & 117 & 36 & 81 & & 76 & 36 & 40 & \\
\hline $\mathrm{N}$ stage & & & & 0.958 & & & & 0.457 \\
\hline NO & 93 & 40 & 53 & & 63 & 29 & 34 & \\
\hline $\mathrm{N}+$ & 210 & 91 & 119 & & 159 & 82 & 77 & \\
\hline TNM stage & & & & 0.006 & & & & 0.485 \\
\hline ૫ & 30 & 18 & 12 & & 19 & 7 & 12 & \\
\hline ૫ & 118 & 59 & 59 & & 97 & 50 & 47 & \\
\hline$\square$ & 155 & 54 & 101 & & 106 & 54 & 52 & \\
\hline
\end{tabular}

SIl systemic immune-inflammation index, ESCC esophageal squamous cell carcinoma, PSM propensity score matching

\section{SIl and survival in all patients}

Overall, the 1-, 3- and 5-year OS rates were $74.9 \%, 35.5 \%$ and $25.0 \%$, respectively. The 1-, 3- and 5-year PFS rates were $57.1 \%$, $27.6 \%$ and $19.9 \%$, respectively. For the patients with a high SII $(n=172)$, the 1 -, 3 - and 5 -year OS rates were $65.7 \%, 28.4 \%$ and $18.7 \%$, respectively. The corresponding rates in patients with a low SII $(n=131)$ were $87.0 \%, 44.8 \%$ and $33.2 \%$, respectively. In the high SII group and the low SII group, the 1-, 3-, 5-year PFS rates were 46.5\%, 20.1\%, 12.8\% and 71.0\%, 37.3\%, 29.2\%, respectively. Kaplan-Meier analysis showed that overall the high SII group had poorer OS and PFS compared with the low SII group (Fig. 1).

\section{Univariate and multivariate survival analysis in all patients}

In univariate analyses, the age, tumor location, T stage, lymph node metastasis, irradiation method, radiotherapy dose, adjuvant chemotherapy and the SII level were significantly associated with OS and PFS. Multivariate analyses identified the T stage, lymph node metastasis, adjuvant chemotherapy and SII as independent prognostic factors for OS (Table 2) and PFS (Table 3).

Table 2 Univariate and multivariate analyses of prognostic factors for OS in 303 ESCC patients 


\begin{tabular}{|c|c|c|c|c|c|c|}
\hline \multirow[t]{2}{*}{ Variables } & \multicolumn{3}{|c|}{ Univariate analysis } & \multicolumn{3}{|c|}{ Multivariate analysis } \\
\hline & $P$ & HR & $95 \% \mathrm{Cl}$ & $P$ & HR & $95 \% \mathrm{Cl}$ \\
\hline Sex (male/female) & 0.979 & 1.003 & $0.776 \sim 1.298$ & & & \\
\hline $\operatorname{Age}(\leq 65 />65)$ & 0.016 & 1.378 & $1.062 \sim 1.789$ & 0.467 & 1.116 & $0.830 \sim 1.502$ \\
\hline Tumor location (cervical,upper/middle,lower) & 0.006 & 1.481 & $1.121 \sim 1.956$ & 0.826 & 1.035 & $0.764 \sim 1.402$ \\
\hline T stage(T1,T2/T3,T4) & $<0.001$ & 2.057 & $1.516 \sim 2.792$ & 0.001 & 1.733 & $1.268 \sim 2.366$ \\
\hline Lymph node metastasis(negative/positive) & 0.010 & 1.456 & $1.093 \sim 1.940$ & 0.011 & 1.463 & $1.090 \sim 1.964$ \\
\hline irradiation method (IFI/ENI) & $<0.001$ & 0.574 & $0.439 \sim 0.750$ & 0.117 & 0.788 & $0.584 \sim 1.062$ \\
\hline RT dose ( $\leq 60 G y />60 G y)$ & 0.005 & 0.693 & $0.538 \sim 0.893$ & 0.326 & 0.870 & $0.659 \sim 1.149$ \\
\hline Adjuvant chemotherapy (no/yes) & $<0.001$ & 0.611 & $0.474 \sim 0.788$ & 0.016 & 0.701 & $0.524 \sim 0.937$ \\
\hline SII $(\leq 586 />586)$ & $<0.001$ & 1.667 & $1.284 \sim 2.163$ & 0.011 & 1.420 & $1.083 \sim 1.860$ \\
\hline
\end{tabular}

OS overall survival, ESCC esophageal squamous cell carcinoma, HR hazard ratio, C/ confidence interval, IF/ involved-field irradiation, EN/ elective node irradiation, $R T$ radiotherapy, S/l systemic immune-inflammation index

Table 3 Univariate and multivariate analyses of prognostic factors for PFS in 303 ESCC patients

\begin{tabular}{|c|c|c|c|c|c|c|}
\hline \multirow[t]{2}{*}{ Variables } & \multicolumn{3}{|c|}{ Univariate analysis } & \multicolumn{3}{|c|}{ Multivariate analysis } \\
\hline & $P$ & HR & $95 \% \mathrm{Cl}$ & $P$ & HR & $95 \% \mathrm{Cl}$ \\
\hline Sex (male/female) & 0.781 & 1.037 & $0.805 \sim 1.334$ & & & \\
\hline $\operatorname{Age}(\leq 65 />65)$ & 0.034 & 1.316 & $1.020 \sim 1.697$ & 0.563 & 1.088 & $0.818 \sim 1.445$ \\
\hline Tumor location (cervical,upper/middle,lower) & 0.013 & 1.409 & $1.075 \sim 1.848$ & 0.905 & 0.982 & $0.730 \sim 1.321$ \\
\hline T stage(T1,T2/T3,T4) & $<0.001$ & 1.947 & $1.448 \sim 2.617$ & 0.001 & 1.642 & $1.212 \sim 2.225$ \\
\hline Lymph node metastasis(negative/positive) & 0.005 & 1.489 & $1.124 \sim 1.971$ & 0.006 & 1.492 & $1.120 \sim 1.988$ \\
\hline irradiation method (IFI/ENI) & $<0.001$ & 0.566 & $0.436 \sim 0.735$ & 0.065 & 0.758 & $0.564 \sim 1.018$ \\
\hline RT dose ( $\leq 60 \mathrm{~Gy} />60 \mathrm{~Gy})$ & 0.006 & 0.707 & $0.552 \sim 0.907$ & 0.411 & 0.891 & $0.676 \sim 1.174$ \\
\hline Adjuvant chemotherapy (no/yes) & $<0.001$ & 0.630 & $0.491 \sim 0.809$ & 0.025 & 0.726 & $0.549 \sim 0.961$ \\
\hline SII $(\leq 586 />586)$ & $<0.001$ & 1.658 & $1.284 \sim 2.140$ & 0.012 & 1.411 & $1.079 \sim 1.845$ \\
\hline
\end{tabular}

PFS progression-free survival, ESCC esophageal squamous cell carcinoma, $H R$ hazard ratio, Cl confidence interval, IFI involvedfield irradiation, EN/ elective node irradiation, $R T$ radiotherapy, S/l systemic immune-inflammation index

\section{Changes of Sll and survival in all patients}

Based on the SII values before-, during-, and after the radiotherapy, the box plot showed the increasing trend of SII. Based on weekly averages for SII, the fitting curve and the function $\left(Y=787 \mathrm{e}^{0.197 x}, P<0.001\right)$ indicated that the mean SII displayed an exponential increase trend during radiotherapy (Fig. 2).

The median of SII after radiotherapy was 1457 . On the basis of SII after radiotherapy, the two groups were divided into four subgroups: high-high (baseline SII > 586 and SII after radiotherapy > 1457); high-low (baseline SII > 586 and SII after radiotherapy S 1457); low-high (baseline SII $\leq 586$ and SII after radiotherapy > 1457); and low-low (baseline SII $\leq 586$ and SII after radiotherapy $\leq 1457$ ). Table 4 showed the relationships between changes of SIl and survival according to these four groups. 
Table 4 Relationships between changes of SII and survival in 303 ESCC patients

\begin{tabular}{|c|c|c|c|c|c|c|c|c|}
\hline \multirow{2}{*}{$\begin{array}{l}\text { Baseline } \\
\text { SII }\end{array}$} & \multirow{2}{*}{$\begin{array}{l}\text { SII } \\
\text { after } \\
\text { RT }\end{array}$} & \multirow[t]{2}{*}{$\mathbf{N}$} & \multicolumn{3}{|l|}{ Overall survival } & \multicolumn{3}{|c|}{ Progression-free survival } \\
\hline & & & Median $(95 \% \mathrm{Cl})$ & $\mathrm{HR}(95 \% \mathrm{Cl})$ & $P$ & $\begin{array}{l}\text { Median } \\
(95 \% \mathrm{Cl})\end{array}$ & $\mathrm{HR}(95 \% \mathrm{Cl})$ & $P$ \\
\hline High & High & 108 & $\begin{array}{l}14.7 \\
(12.0 \sim 17.3)\end{array}$ & 1 & - & $10.2(7.8 \sim 12.5)$ & 1 & - \\
\hline High & Low & 64 & $\begin{array}{l}21.2 \\
(14.9 \sim 27.4)\end{array}$ & $\begin{array}{l}0.841 \\
(0.604 \sim 1.171)\end{array}$ & 0.306 & $\begin{array}{l}12.6 \\
(4.8 \sim 20.3)\end{array}$ & $\begin{array}{l}0.841 \\
(0.604 \sim 1.171)\end{array}$ & 0.306 \\
\hline Low & High & 43 & $\begin{array}{l}33.1 \\
(23.2 \sim 43.1)\end{array}$ & $\begin{array}{l}0.515 \\
(0.341 \sim 0.778)\end{array}$ & 0.002 & $\begin{array}{l}23.4 \\
(12.2 \sim 34.6)\end{array}$ & $\begin{array}{l}0.515 \\
(0.341 \sim 0.778)\end{array}$ & 0.002 \\
\hline Low & Low & 88 & $32.1(19.6 \sim 44.5)$ & $\begin{array}{l}0.587 \\
(0.430 \sim 0.803)\end{array}$ & 0.001 & $\begin{array}{l}25.0 \\
(17.8 \sim 32.1)\end{array}$ & $0.587(0.430 \sim 0.803)$ & 0.001 \\
\hline
\end{tabular}

S/l systemic immune-inflammation index, ESCC esophageal squamous cell carcinoma, $R T$ radiotherapy, $C /$ confidence interval, $H R$ hazard ratio

\section{SIl and survival in propensity score matched patients}

Considered the tumor location, T stage and TNM stage were imbalance between high SIl group and low SII group (Table 1), we applied a 1:1 ratio propensity score matching (PSM). After PSM, there were 222 patients in all, and the clinicopathological characteristics were balanced and evenly distributed between two groups ( $P>0.05$, Table 1$)$. The Kaplan-Meier survival curves in the matched patients showed that the high SII group had poorer OS and PFS compared with the low SII group (Fig. 3).

\section{Univariate and multivariate survival analysis in propensity score matched patients}

A univariate analysis revealed that the age, T stage, irradiation method, radiotherapy dose, adjuvant chemotherapy and SII were significant predictors of OS. In multivariate analysis, the T stage, irradiation method and SII were identified as independent prognostic factors for OS (Table 5).

Univariate analysis identified the T stage, irradiation method, radiotherapy dose, adjuvant chemotherapy and SII as significant risk factors for PFS. In the multivariate analysis, the T stage, irradiation method, adjuvant chemotherapy and SII were conformed as independent prognostic factors for PFS (Table 6).

Table 5 Univariate and multivariate analyses of prognostic factors for OS in propensity score matched 222 ESCC patients

\begin{tabular}{|c|c|c|c|c|c|c|}
\hline \multirow[t]{2}{*}{ Variables } & \multicolumn{3}{|c|}{ Univariate analysis } & \multicolumn{3}{|c|}{ Multivariate analysis } \\
\hline & $P$ & HR & $95 \% \mathrm{Cl}$ & $P$ & HR & $95 \% \mathrm{Cl}$ \\
\hline Sex (male/female) & 0.601 & 1.082 & $0.806 \sim 1.452$ & & & \\
\hline $\operatorname{Age}(\leq 65 />65)$ & 0.029 & 1.395 & $1.035 \sim 1.881$ & 0.601 & 1.095 & $0.779 \sim 1.540$ \\
\hline Tumor location (cervical,upper/middle,lower) & 0.591 & 1.095 & $0.788 \sim 1.521$ & & & \\
\hline T stage(T1,T2/T3,T4) & $<0.001$ & 1.948 & $1.389 \sim 2.733$ & $<0.001$ & 2.322 & $1.634 \sim 3.300$ \\
\hline Lymph node metastasis(negative/positive) & 0.232 & 1.223 & $0.879 \sim 1.700$ & & & \\
\hline irradiation method (IFI/ENI) & $<0.001$ & 0.518 & $0.380 \sim 0.706$ & 0.026 & 0.689 & $0.496 \sim 0.957$ \\
\hline RT dose ( $\leq 60 \mathrm{~Gy} />60 \mathrm{~Gy})$ & 0.005 & 0.658 & $0.492 \sim 0.879$ & 0.090 & 0.767 & $0.564 \sim 1.042$ \\
\hline Adjuvant chemotherapy (no/yes) & $<0.001$ & 0.540 & $0.403 \sim 0.724$ & 0.051 & 0.710 & $0.503 \sim 1.001$ \\
\hline SII $(\leq 586 />586)$ & $<0.001$ & 1.893 & $1.415 \sim 2.533$ & $<0.001$ & 2.222 & $1.634 \sim 3.021$ \\
\hline
\end{tabular}


OS overall survival, ESCC esophageal squamous cell carcinoma, HR hazard ratio, C/ confidence interval, IFl involved-field irradiation, $E N /$ elective node irradiation, $R T$ radiotherapy, SI/ systemic immune-inflammation index

Table 6 Univariate and multivariate analyses of prognostic factors for PFS in propensity score matched 222 ESCC patients

\begin{tabular}{|c|c|c|c|c|c|c|}
\hline \multirow[t]{2}{*}{ Variables } & \multicolumn{3}{|c|}{ Univariate analysis } & \multicolumn{3}{|c|}{ Multivariate analysis } \\
\hline & $P$ & HR & $95 \% \mathrm{Cl}$ & $P$ & HR & $95 \% \mathrm{Cl}$ \\
\hline Sex (male/female) & 0.565 & 1.088 & $0.816 \sim 1.450$ & & & \\
\hline $\operatorname{Age}(\leq 65 />65)$ & 0.070 & 1.306 & $0.979 \sim 1.744$ & & & \\
\hline Tumor location (cervical,upper/middle,lower) & 0.916 & 0.983 & $0.716 \sim 1.350$ & & & \\
\hline T stage $(\mathrm{T} 1, \mathrm{~T} 2 / \mathrm{T} 3, \mathrm{~T} 4)$ & $<0.001$ & 1.888 & $1.363 \sim 2.617$ & $<0.001$ & 2.155 & $1.537 \sim 3.021$ \\
\hline Lymph node metastasis(negative/positive) & 0.225 & 1.218 & $0.886 \sim 1.675$ & & & \\
\hline irradiation method (IFI/ENI) & $<0.001$ & 0.536 & $0.398 \sim 0.723$ & 0.028 & 0.699 & $0.508 \sim 0.962$ \\
\hline RT dose ( $\leq 60 \mathrm{~Gy} />60 \mathrm{~Gy})$ & 0.003 & 0.657 & $0.495 \sim 0.871$ & 0.073 & 0.763 & $0.567 \sim 1.026$ \\
\hline Adjuvant chemotherapy (no/yes) & $<0.001$ & 0.571 & $0.430 \sim 0.758$ & 0.027 & 0.714 & $0.530 \sim 0.963$ \\
\hline SII $(\leq 586 />586)$ & $<0.001$ & 1.656 & $1.247 \sim 2.198$ & $<0.001$ & 1.865 & $1.384 \sim 2.515$ \\
\hline
\end{tabular}

PFS progression-free survival, ESCC esophageal squamous cell carcinoma, $H R$ hazard ratio, $C /$ confidence interval, IF/ involvedfield irradiation, EN/ elective node irradiation, $R T$ radiotherapy, SI/ systemic immune-inflammation index

\section{Discussion}

During the past decade, there has been an increasing interest in the associations between systemic inflammation and outcomes of several malignancies [5-7]. However, just few studies regarding SII in patients with ESCC undergoing radical radiotherapy are available. In the present study, 303 ESCC patients received radical radiotherapy were analyzed retrospectively. The results showed that elevated SII was associated with tumor location, T stage and TNM stage. The OS and PFS were poorer in the high SII group than in the low SII group. SIl was an independent prognostic factor for OS and PFS in ESCC patients treated with radical radiotherapy. To the best of our knowledge, this is the first report focused on the change trend of SII and the associations between change of SII and survival. The results showed that SII increased significantly during radiotherapy, and the change of SII was associated with OS and PFS. Moreover, it was worth mentioning that PSM was applied in the present study to reduce biases due to the different distributions of co-variables among the comparable groups and to increase statistical power. In 222 propensity matched ESCC patients, SII retained prognostic significance for OS and PFS.

Many studies have described the role of preoperative SII in the prognosis of various malignancies. SII has been demonstrated to be associated with postoperative survival in colorectal cancer, lung cancer, breast cancer, hepatocellular carcinoma, et al [12-15]. Moreover, in esophageal carcinoma, the preoperative SIl was confirmed to be an independent predictor for survival and quality of life. Geng et al. [16] initially reported prognostic value of SII in patients with ESCC. Based on the retrospective study of 916 patients with ESCC who underwent radical surgery, they demonstrated that SII was an independent risk factor for OS and the prognostic value of SII is superior to PLR, NLR and MLR. Wang et al. [17] reported that higher SIl was associated with advanced T stage, N stage and TNM stage, and the preoperative SII was a promising biomarker for predicting survival and quality of life of patients with ESCC. Similar results were shown in another study. Gao et al. [20] retrospectively analyzed 468 ESCC patients who underwent curative esophagectomy with R0 resection. Their result showed that a high SIl was an independent predictor for both OS and DFS in patients with surgically resected ESCC, and by comparing the areas under the AUC curve, SII was superior to NLR, PLR, and MLR in terms of prognostic ability. Undoubtedly, our results in ESCC patients undergoing radical radiotherapy were consistent with previous reports in ESCC patients undergoing curative esophagectomy. However, we found that in our study, the optimal cutoff value of SII was higher, and the OS was lower than that in previous studies. One possible reason was that the 
patients undergoing radical radiotherapy had more advanced TNM stage than patients undergoing surgery. As previously mentioned, elevated SII was associated with advanced TNM stage [17], and TNM stage was one of prognostic factors for OS [20].

The mechanism of the correlation between SII and overall survival in patients with esophageal cancer remains unclear. Several potential theories may be used to explain the prognostic values of SII. Firstly, the cancer-mediated generation of bone marrow cells and various cytokines released during the generation may promote tumor angiogenesis, invasion and metastasis, shield cancer cells from immune destruction, and induce resistance to cytotoxic drugs [21]. Secondly, neutrophils can release cytokines leading to $\mathrm{T}$ cell activation disorders [22]. Platelets and neutrophils promote adhesion and implantation of distant organs by secreting vascular endothelial growth factor $[23,24]$. Thirdly, lymphocytes display a significant antitumor role by inducing cytotoxic cell apoptosis and inhibiting tumor cell proliferation and migration, instituting the host's immune response to cancer.[25, 26] Therefore, inflammation deeply influences cancer microenvironment that supports cancer progression. Based on the above theories, SII should be an objective marker that reflects the balance between host inflammatory and immune response status, and a promising prognostic predictor for cancer patients.

There are several potential limitations in this study. First, this is a retrospective, single-institute study, and there may be selection bias during patients collection, although the PSM has been applied to imbalance the baseline characteristics. Second, some of our results were consistent with previous reports, but the optimal cutoff value of SII was different with previous similar studies. So it was not easy to verify our conclusions in another independent cohort. Therefore, larger prospective studies are required in the future to confirm these preliminary results.

In conclusion, the SII is a significant and independent predictor for survival of ESCC patients undergoing radical radiotherapy. SII displayed an exponential increase trend during radiotherapy. The change of SII was also associated with OS and PFS. Based on simple and inexpensive standard laboratory measurements, SII can be a promising marker for ESCC patients.

\section{Abbreviations}

SII: systemic immune-inflammation index; ESCC: esophageal squamous cell carcinoma ; PSM: propensity score matching; OS: overall survival; PFS: progression-free survival; PLR: platelet-lymphocyte ratio; NLR: neutrophil-lymphocyte ratio; MLR: monocytelymphocyte ratio; AJCC: American Joint Committee on cancer; GTV: gross tumor volume; ROC: receiver operating characteristics; HR: hazard ratio; Cl: confidence interval; IFI: involved-field irradiation; ENI: elective node irradiation; RT: radiotherapy.

\section{Declarations}

\section{Ethics approval and consent to participate}

This study was approved by the Ethics Committee of Fourth Affiliated Hospital of Hebei Medical University. Written informed consent was obtained from each participant by the institutional guidelines.

\section{Consent for publication}

Informed consent was obtained from all participants for publication.

\section{Availability of data and materials}

All data included in the present study were presented in the main manuscript.

\section{Competing interests}

The authors declare that they have no competing interests.

\section{Funding}

This study was supported by Health Commission of Hebei Province (Grant No. 20190725). 


\section{Authors' contributions}

YZ: data acquisition and manuscript writing, CYS and JRX: data acquisition and data analysis, SGL, WZD and JL: follow up and quality control of data, WBS: manuscript review, SCZ: study concept and design. All authors read and approved the final manuscript.

\section{Acknowledgements}

We thank the patients for their time and participation in this study.

\section{References}

1. Crusz SM, Balkwill FR. Inflammation and cancer: advances and new agents [J]. Nat Rev Clin Oncol. 2015; 12: 584-96.

2. Lin Y, Totsuka Y, He Y, Kikuchi S, Qiao Y, Ueda J, Tanaka H. (2013). Epidemiology of esophageal cancer in Japan and China. Journal of Epidemiology, 23(4), 233-242.

3. Franco AT, Corken A, Ware J. Platelets at the interface of thrombosis, inflammation, and cancer. Blood. 2015; 126: 582-8.

4. Singel KL, Segal BH. Neutrophils in the tumor microenvironment: trying to heal the wound that cannot heal [J]. Immunol Rev. 2016; 273: 329-43.

5. Liu X, Chen S, Liu J, Xu D, Li W, Zhan Y, et al. Impact of systemic inflammation on gastric cancer outcomes. PLoS One. 2017; 12(3): e0174085.

6. Chen X, Zhang L, Liu X. Association of systemic inflammation with survival in EGFR wild-type lung cancer. Ann Oncol. 2018; 29 Suppl 9: ix155.

7. Tuomisto AE, Mäkinen MJ, Väyrynen JP. Systemic inflammation in colorectal cancer: Underlying factors, effects, and prognostic significance. World J Gastroenterol. 2019; 25(31): 4383-4404.

8. Yodying H, Matsuda A, Miyashita M, Matsumoto S, Sakurazawa N, Yamada M, et al. Prognostic significance of neutrophil-tolymphocyte ratio and platelet-to-lymphocyte ratio in oncologic outcomes of esophageal cancer: a systematic review and meta-analysis. Ann Surg Oncol.2016; 23(2): 646-54.

9. Deng J, Zhang P, Sun Y, Peng P, Huang Y. Prognostic and clinicopathological significance of platelet to lymphocyte ratio in esophageal cancer: a meta-analysis. J Thorac Dis.2018; 10(3): 1522-1531.

10. Mandaliya H, Jones M, Oldmeadow C, Nordman II. Prognostic biomarkers in stage IV non-small cell lung cancer (NSCLC): neutrophil to lymphocyte ratio (NLR), lymphocyte to monocyte ratio (LMR), platelet to lymphocyte ratio (PLR) and advanced lung cancer inflammation index (ALI). Transl Lung Cancer Res. 2019; 8(6): 886-894.

11. Gaballah A, Hussein T, Ezzat A, Magdy M. Pre-treatment peripheral neutrophil-lymphocyte ratio as a prognostic factor in gastric cancer. Ann Oncol. 2018; 29 Suppl 5: v15.

12. Wang BL, Tian L, Gao XH, Ma XL, Wu J, Zhang CY, et al. Dynamic change of the systemic immune inflammation index predicts the prognosis of patients with hepatocellular carcinoma after curative resection. Clin Chem Lab Med. 2016; 54(12): 1963-1969.

13. Chen JH, Zhai ET, Yuan YJ, Wu KM, Xu JB, Peng JJ, et al. Systemic immune-inflammation index for predicting prognosis of colorectal cancer. World journal of gastroenterology. 2017; 23: 6261.

14. Shoji F. Clinical impact of the systemic immune-inflammation index in non-small cell lung cancer patients. Ann Transl Med. 2020; 8(11): 668.

15. Hua X, Long ZQ, Zhang YL, Wen W, Guo L, Xia W, et al. Prognostic value of preoperative systemic immune-inflammation index in breast cancer: a propensity score-matching study. Front Oncol.2020; 10: 580.

16. Geng Y, Shao Y, Zhu D, Zheng X, Zhou Q, Zhou W, et al. Systemic immune-inflammation index predicts prognosis of patients with esophageal squamous cell carcinoma: a propensity score-matched analysis. Scientific reports. 2016; 6: 39482.

17. Wang L, Wang C, Wang J, Huang X, Cheng Y. A novel systemic immune-inflammation index predicts survival and quality of life of patients after curative resection for esophageal squamous cell carcinoma. Journal of cancer research and clinical oncology. 2017; 143: 2077-86. 
18. Feng J-F, Chen S, Yang X. Systemic immune-inflammation index (SII) is a useful prognostic indicator for patients with squamous cell carcinoma of the esophagus. Medicine. 2017; 96.

19. Zhang H, Shang X, Ren P, Gong L, Ahmed A, Ma Z, et al. The predictive value of a preoperative systemic immuneinflammation index and prognostic nutritional index in patients with esophageal squamous cell carcinoma. Journal of Cellular Physiology. 2019; 234: 1794-802.

20. Gao Y, Guo W, Cai S, Zhang F, Shao F, Zhang G, et al. Systemic immune-inflammation index (SII) is useful to predict survival outcomes in patients with surgically resected esophageal squamous cell carcinoma. J Cancer. 2019; 10(14): 3188-3196.

21. Diakos Cl., Charles KA, McMillan DC, Clarke SJ. Cancer-related inflammation and treatment effectiveness [J]. Lancet Oncol. $2014 ; 15$, e493-503.

22. Muller I, Munder M, Kropf P, Hänsch GM. Polymorphonuclear neutrophils and T lymphocytes: strange bedfellows or brothers in arms? [J] Trends Immunol. 2009; 30: 522-530.

23. Hu B, Yang X, Xu Y, Sun YF, Sun C, Guo W, et al. Systemic immune-inflammation index predicts prognosis of patients after curative resection for hepatocellular carcinoma [J]. Clin Cancer Res. 2014; 20: 6212-6222.

24. Cools-Lartigue J, Spicer J, McDonald B, Gowing S, Chow S, Giannias B, et al. Neutrophil extracellular traps sequester circulating tumor cells and promote metastasis [J]. J Clin Invest. 2013. DOI: 10.1172/JCl67484.

25. De Giorgi U, Mego M, Scarpi E, Giuliano M, Giordano A, Reuben JM, et al. Relationship between lymphocytopenia and circulating tumor cells as prognostic factors for overall survival in metastatic breast cancer. Clin Breast Cancer 2012; 12: 2649.

26. Minami T, Minami T, Shimizu N, Yamamoto Y, De Velasco M, Nozawa M, et al. Identification of programmed death ligand 1derived peptides capable of inducing cancerreactive cytotoxic T lymphocytes from HLA-A24+ patients with renal cell carcinoma. J Immunother. 2015; 38: 285-91.

\section{Figures}
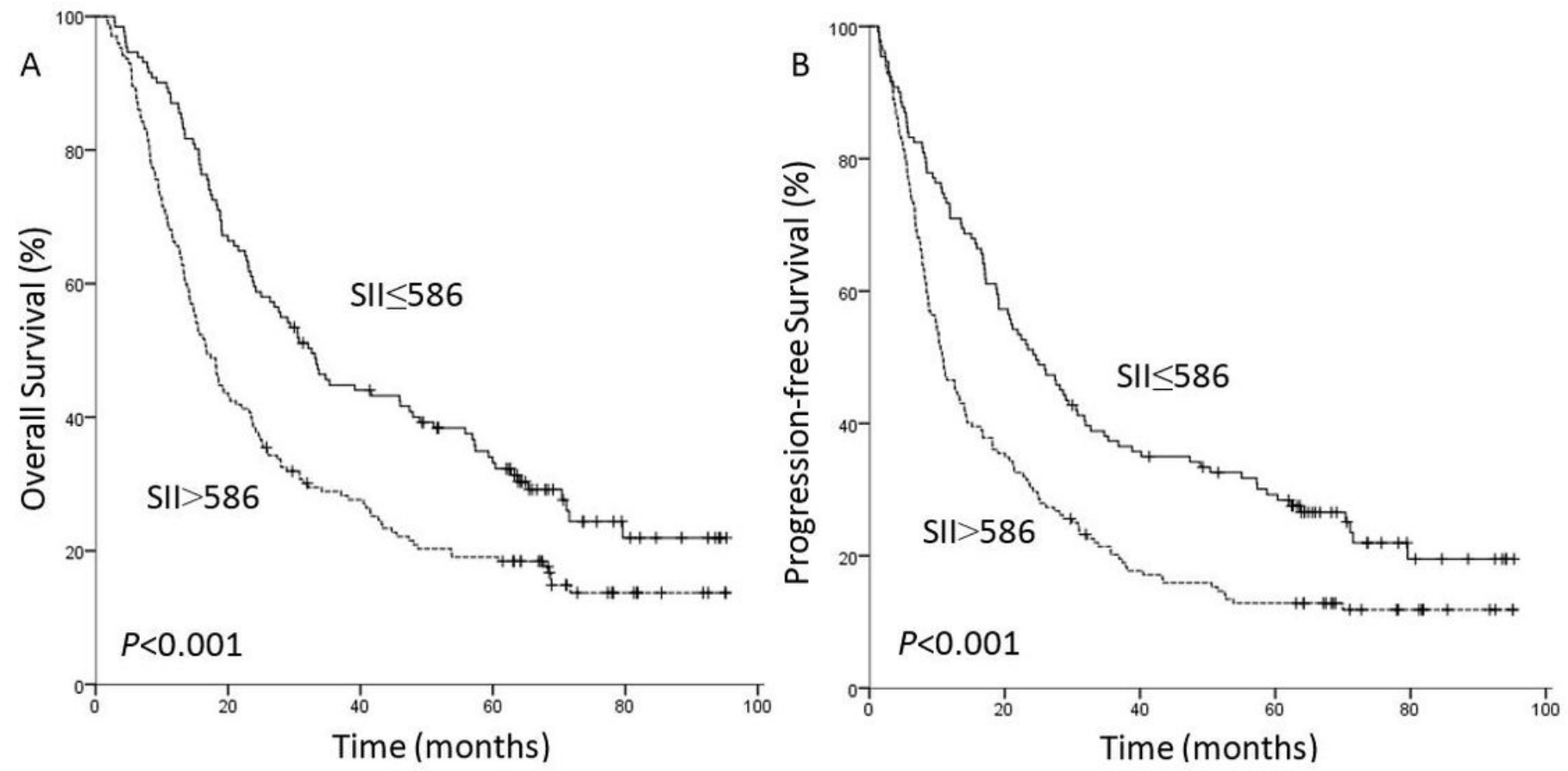

\section{Figure 1}

Kaplan-Meier curves of survival based on SII in 303 ESCC patients undergoing definitive radiotherapy. A: overall survival; B: progression-free survival. 

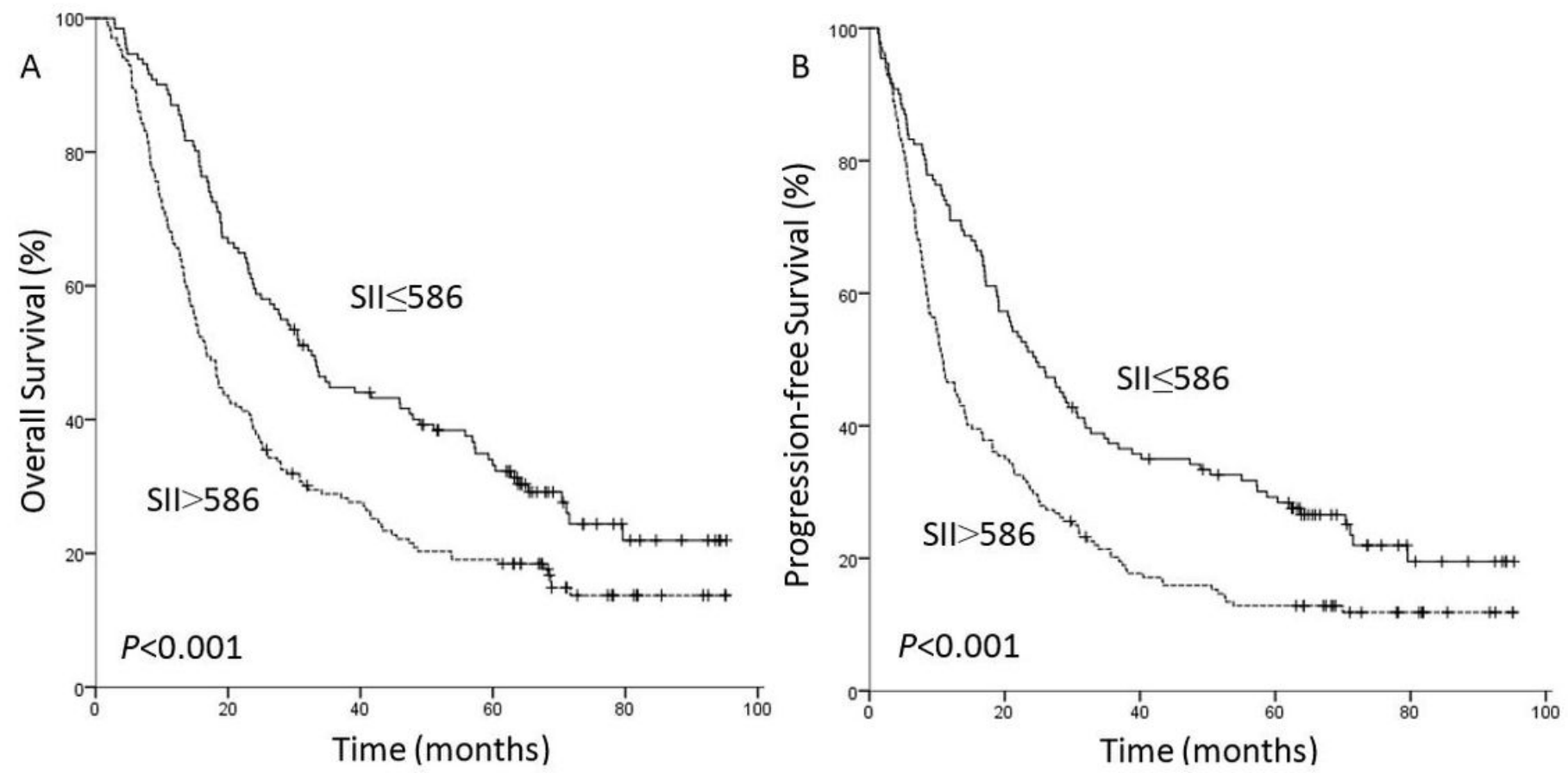

Figure 1

Kaplan-Meier curves of survival based on SII in 303 ESCC patients undergoing definitive radiotherapy. A: overall survival; B: progression-free survival.
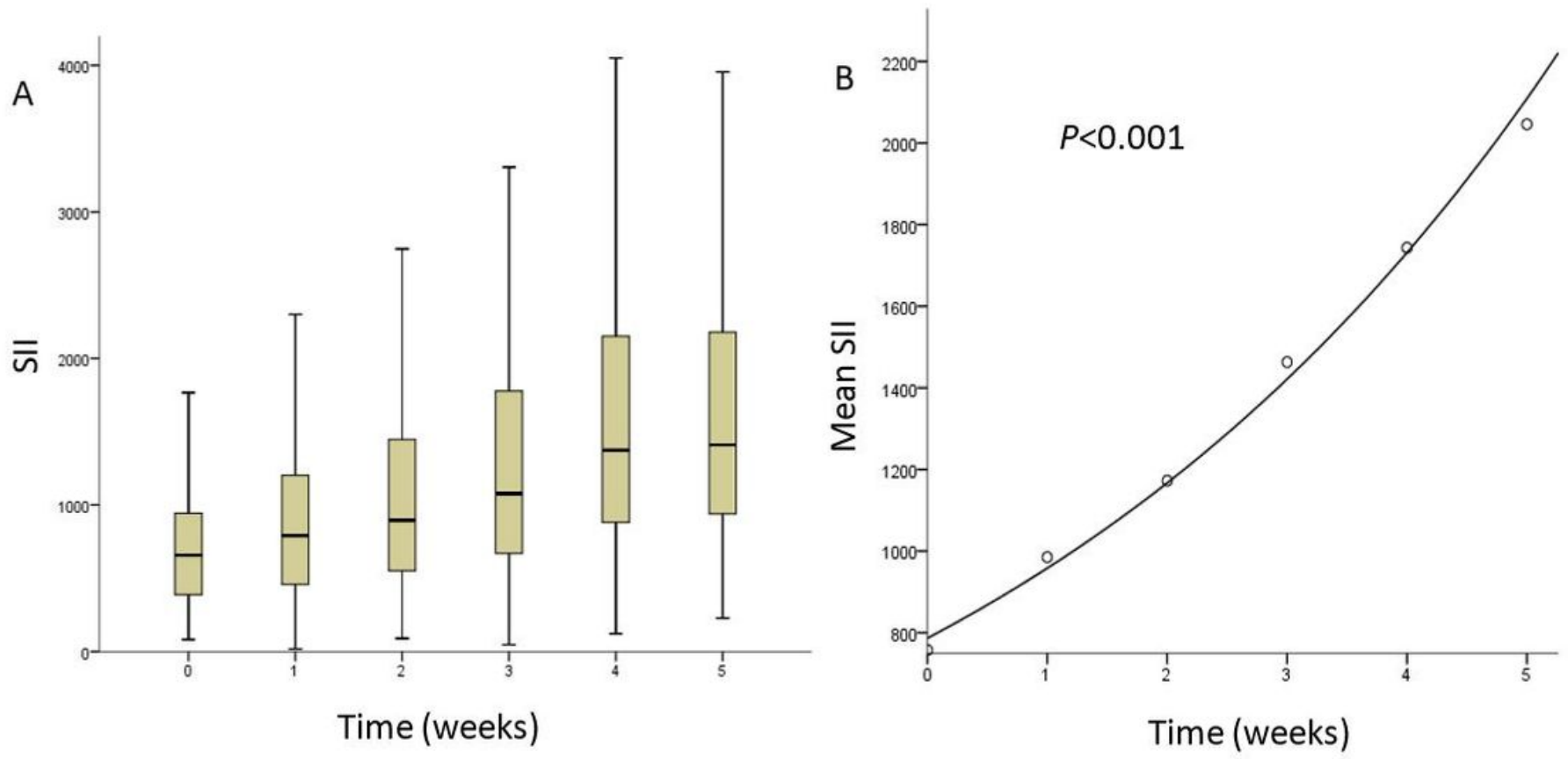

Figure 2

The change trend of SIl during radiotherapy in 303 ESCC patients (A: the box plot of SIl during radiotherapy; $\mathrm{B}$ : the change trend of mean SII) 

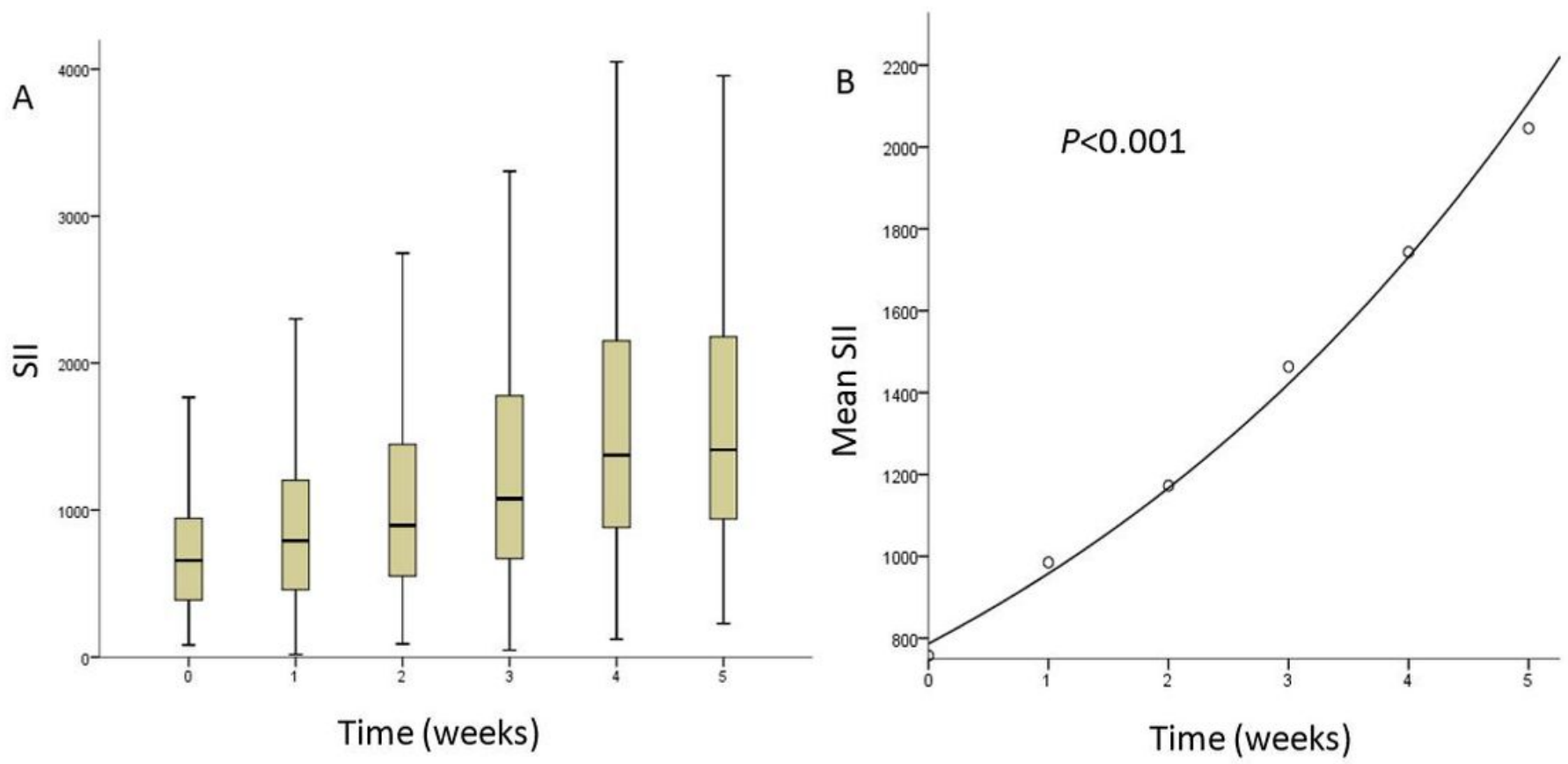

Figure 2

The change trend of SII during radiotherapy in 303 ESCC patients (A: the box plot of SIl during radiotherapy; $\mathrm{B}$ : the change trend of mean SII)
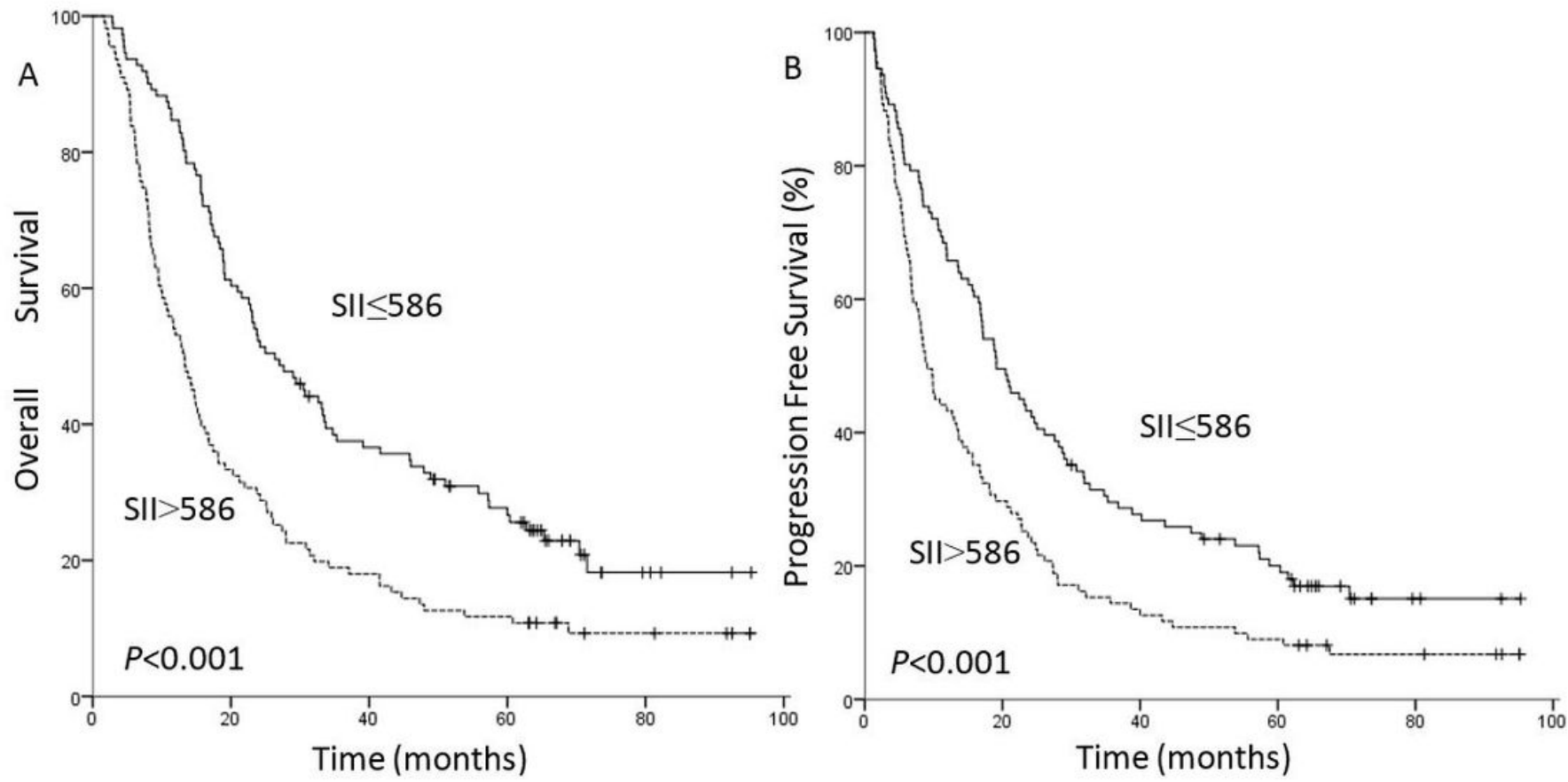

\section{Figure 3}

Kaplan-Meier curves of survival based on SII in propensity score matched 222 ESCC patients undergoing definitive radiotherapy. A: overall survival; B: progression-free survival. 

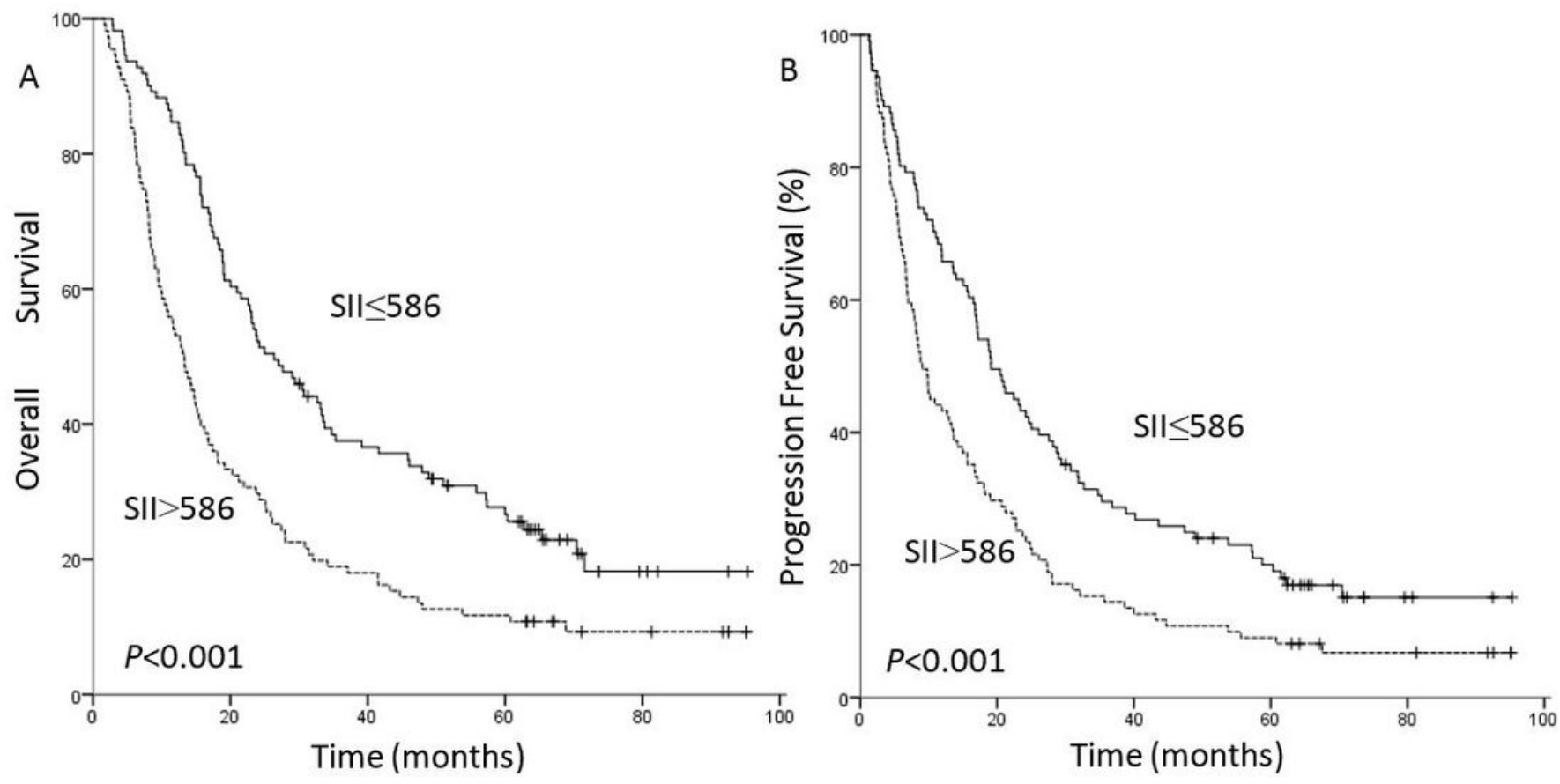

Figure 3

Kaplan-Meier curves of survival based on SII in propensity score matched 222 ESCC patients undergoing definitive radiotherapy. A: overall survival; B: progression-free survival. 